\title{
The signals of FGFs on the neurogenesis of embryonic stem cells
}

\author{
Ching-Wen Chen', Chin-San Liu², Ing-Ming Chiu³, Shih-Cheng Shen', Hung-Chuan Pan4, Kun-Hsiung Lee5 , Shinn- \\ Zong Lin ${ }^{6}$ and Hong-Lin Su*1,7
}

\begin{abstract}
Background: Neural induction is a complex process and the detailed mechanism of FGF-induced neurogenesis remains unclear.

Methods: By using a serum-free neural induction method, we showed that FGF1 dose-dependently promoted the induction of Sox1/N-cadherin/nestin triple positive cells, which represent primitive neuroblasts, from mouse embryonic stem (ES) cells.

Results: We demonstrated that FGF1, FGF2, and FGF4, but not FGF8b, enhanced this neurogenesis. Especially, FGFenhanced neurogenesis is not mediated through the rescue of the apoptosis or the enhancement of the proliferation of Sox $1+$ cells. We further indicated that the inactivation of c-Jun N-terminal kinase-1 (JNK-1) and extracellular signalrelated kinase-2 (ERK-2), but not p38 mitogen-activated protein kinase (MAPK), inhibited the neural formation through the inhibition of ES differentiation, but not through the formation of endomesodermal cells.
\end{abstract}

Conclusions: These lines of evidence delineated the roles of FGF downstream signals in the early neural differentiation of ES cells.

\section{Background}

In the early gastrula of the chicken, temporary treatment of the primitive ectoderm with Hensen's node for 5 hours steers the ectoderm to become the neural fate [1,2]. FGF was shown to be responsible for this instructive ability of node and for the maintenance of later neural instructive signals [3,4]. FGF first activates ERNI during early gastrulation and consequently triggers the zinc-finger transcriptional activator, Churchill, and its downstream target Sip1 in late gastrulation [4]. In Xenopus, the study of neural induction has revealed the essential role of Ras/MAPK activation for neurogenesis in uncommitted ectoderm and in dissociated animal cap cells, suggesting that the requirement of FGF signals in neural induction is conserved in chordates [5].

ES cells, which resemble epiblast cells in the blastocyst, provide an alternative approach to the study of early development in mammals [6,7]. Several one-step neural induction models have been established. Trans-retinoic

* Correspondence: suhonglin@gmail.com

1 Department of Life Sciences, National Chung-Hsing University, Taichung, Taiwan

Full list of author information is available at the end of the article acid (RA), a pro-neural inducer, enriches the neural population in a serum-containing embryoid bodies (EBs) system $[8,9]$. However, RA treatment has several drawbacks, including the caudalization of the neural fate, blockage of forebrain induction, and the disruption of normal embryogenesis [9-11]. Co-culture of ES cells with mouse skull-derived stromal cells, such as PA6 cells, or bone marrow-derived cells, such as MS5 cells, efficiently induces the ES cells to become neuron lineages $[8,12]$. However, the factors contributing to this stromal-derived inducing activity are still uncharacterized. ES cells cultured in serum-free Neurobasal medium with N2B27 supplement efficiently differentiate into Sox $1^{+}$neural precursors, which represent the earliest committed neuroblast cells in the developing embryo [13,14]. Specific neuronal subtypes, such as dopaminergic and serotoninergic neurons, are derived from the Sox1 neuroblasts by the addition of defined patterning factors. Although the Neurobasal/N2B27 model provides a simple monoculture differentiation system for ES cells, these cells often undergo apoptosis on days 3 to 5 . Recently, an efficient neural-induction monoculture system with a high sur- 
vival rate for differentiating ES cells was developed and termed as serum-free embryoid bodies formation (SFEB) method [15]. This simple and reproducible system consists of defined components and is suitable for the exploration of downstream FGF signals in the early neurogenesis of mammals.

\section{Methods}

\section{Cell culture and differentiation}

Sox1-GFP knock-in ES cells (46C), from Dr. Austin Smith (University of Cambridge, UK), and ESC 26 cells, were both well-characterized and germline transmissible $[14,16]$. The culture condition of both cells $[14,16]$ and the SFEB method [15] has been described previously in detail.

\section{Reagents}

Human recombinant FGF2, FGF4 and FGF8b were all from R\&D Systems. Recombinant human FGF1 was prepared from Prof. Chiu in Institute of Cell and Systems Medicine, the National Health Research Institutes, Taiwan [17]. Synthetic inhibitors of FGF signaling, including SU5402, LY294002, SB203580, and SP600125, were from Calbiochem; U0126 was purchased from Tocris.

\section{Stable cell establishment}

The plasmid Flag-DsRedT4-NLS was a gift from Tim Shroeder at Helmholtz Center Munich, Institute of Stem Cell Research, Germany. The genes of JNK dominant negative mutants, Flag-JNK1a1apf and Flag-JNK2a2apf $[18,19]$, were obtained from Addgene http:// www.addgene.org and fused with a IRES-DsRed as a reporter. The plasmids were transfected into ES cells with lipofectamine 2000 (Invitrogen). After selection with 0.4

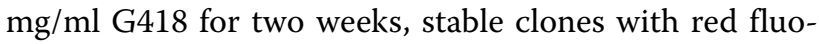
rescence were picked up and maintained with $0.2 \mathrm{mg} / \mathrm{ml}$ G418. The selected ES cells showed normal ES cell morphology and pluripotent gene expression (data not shown).

\section{Immunocytochemistry}

Cells were fixed in $4 \%$ cold paraformaldehyde and permeabilized with $0.3 \%$ Triton-X 100 . Immunocytochemistry was performed with the following primary antibodies: OCT3/4 (1:500, Santa Cruz), Nanog (1:100, Cosmo Bio, Japan), Sox2 (1:4000, Chemicon), N-cadherin (1:100, DSHB, Iowa), FGF receptor 1 (FGFR1) and FGFR3 (both 1:100, Santa Cruz), FGFR2 (1:500, Abcam) and GFP (1:1000, Aves Labs). Images of immunostaining were captured usinga fluorescent microscope (Nikon ECLIPSE 80I) or confocal microscope (LSM510 Meta, Zeiss).

\section{Flow cytometry}

Sox1-GFP ES cells were fully dissociated and analyzed with flow cytometry (FC500, Beckman Coulter). Apopto- sis was measured by staining for Annexin V (AbD Serotec) at room temperature for $10 \mathrm{~min}$ in the dark.

\section{RT-PCR analysis}

Total RNA was isolated from ES cells using REzol ${ }^{\mathrm{mit}} \mathrm{C} \& \mathrm{~T}$ reagent (Protech technology, Taiwan). Primers were applied to detect the expression of FGFR1 (5'-CAC ACT GCC TTC TCC TCC TC-3', 5'-CTC TGC CTC CCT GTC TTC TG-3'), FGFR2 (5'-GGG GAT GTG GAG TTT GTC TG-3', 5'-GCT TCT TGG TCG TGG TCT TC-3'), FGFR3 (5'-CGG CTA CCT GTG AAG TGG AT-3', 5'GCT TGG TCT GTG GGA CTG TT-3'), FGFR4 (5'-AGG AAA TGT GGC TGC TCT TG-3', 5'-GGT GTG TCC AGT AGG GTG CT-3'), Sox1 (5'-CCT CGG ATC TCT GGT CAA GT-3', 5'-TAC AGA GCC GGC AGT CAT AC-3'), and G3PDH (5'-GTG AAG GTC GGT GTG AAC G-3', 5'-GGT GAA GAC ACC AGT AGA CAC TC-3').

\section{Western blot analysis}

ES cells were lysed in RIPA buffer $(50 \mathrm{mM}$ Tris pH7.5, $150 \mathrm{mM} \mathrm{NaCl}, 10 \mathrm{mM}$ EDTA, 1\% NP-40, 0.1\% SDS) plus a cocktail of proteinase inhibitors (Sigma-Aldrich). Denatured proteins were separated by 10\% SDS-PAGE and then transferred to PVDF membranes. Samples were detected with antibodies to ERK1/2, phosphoERK1/2 (pERK1/2), p38 and pp38, JNKs and pJNKs, AKT and pAKT. All MAPK-related antibodies were from Cell Signals and diluted 1:1000 for immunoblotting. Chemiluminescence of immunoreactive bands was detected using secondary horseradish peroxidase-conjugated antibodies (Jackson ImmunoResearch) and ECL reagents (Amersham).

\section{Results}

\section{FGF1 enhanced the generation of Sox $1^{+}$cells from ES cells}

Two germline-transmissible mouse ES cell lines, ESC 26 and Sox1-GFP knock-in cells (46C), were used in this study and the ESC 26 cell was characterized with the expression of pluripotent makers (Fig. 1B to 1D). After dissociation, ES cells were cultured at $2 \times 10^{6}$ cells $/ 10 \mathrm{ml}$ in a defined, serum-free, neural differentiation medium (SFEB method) (Fig. 1A), which is an efficient neural induction method with rare mesendoderm formation [15]. We showed that ES-derived Sox1-GFP+ cell was coexpressed several neural markers, such as nestin, pax6, $\mathrm{N}$-cadherin and Zic1 (Fig. 1E to 1H). In addition, GFAP was not detected in differentiating $46 \mathrm{C}$ cells on day 6 (Fig. $1 \mathrm{I})$, indicating that the Sox $1^{+}$cells under the SFEB culture represented primitive neuroblast cells [15]. Exogenous FGF1, applied from day 1 through day 3, dramatically enhanced the neural induction of ESC26 and 46C cells in a dose-dependent manner, as revealed by the counting of $\mathrm{N}$-cadherin ${ }^{+}$colonies (Fig. $1 \mathrm{~J}$ ) and FACS analysis on day 6 , respectively (Fig. 2A). These results suggest that FGF 


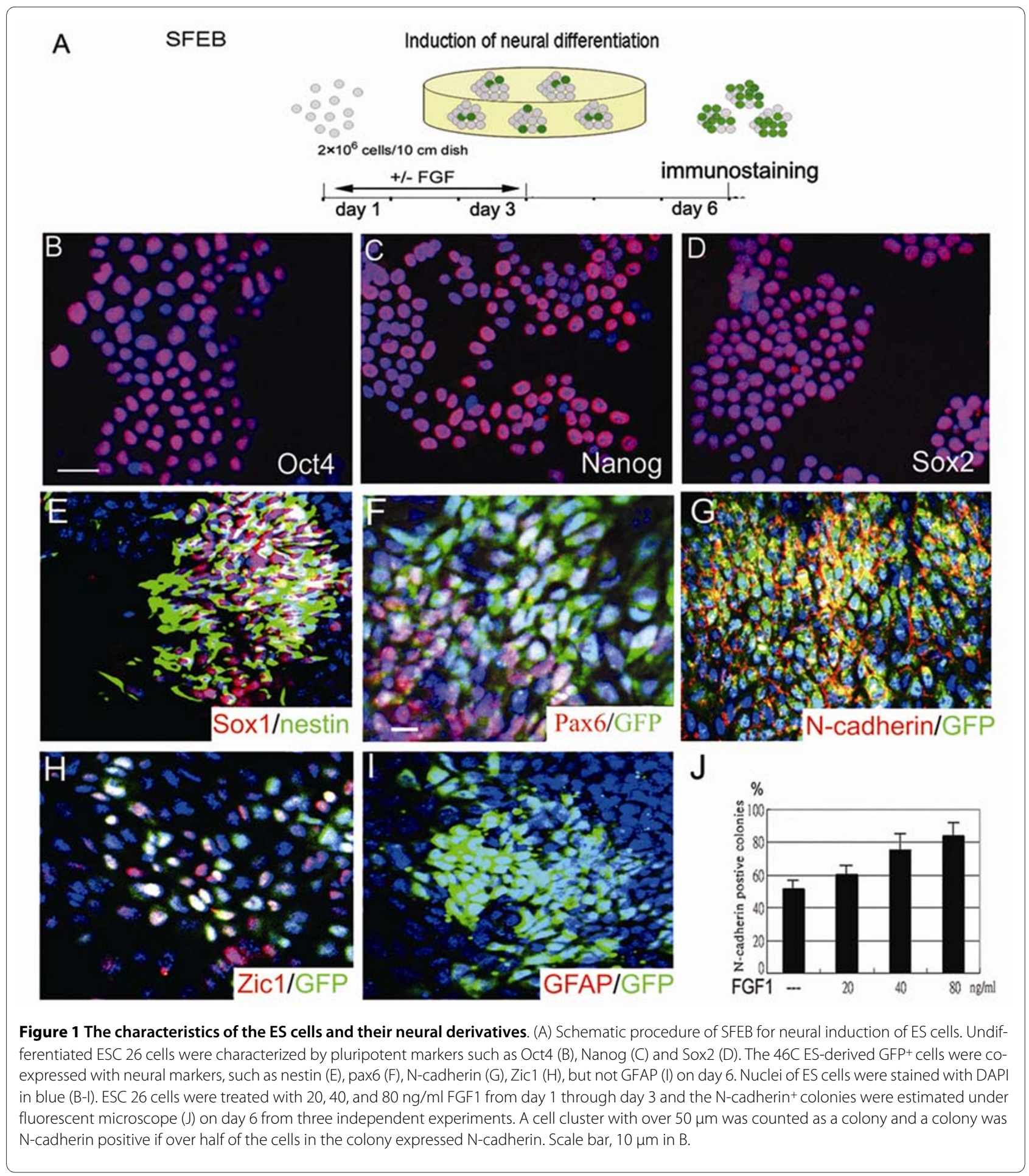

was sufficient to promote the formation of neuroblast cells derived from ES cells.

We next tested the effects of different FGFs on neural formation of ES cells. FGF1, FGF2, and FGF4 all showed significantly elevated neural induction in $46 \mathrm{C}$ cells (Fig. $2 \mathrm{~A})$. However, FGF8b, even at the high concentration of $80 \mathrm{ng} / \mathrm{ml}$, failed to enhance the neural induction of ES cells (Fig. 2A). We further investigated the expression of FGFRs in ES cells during neural induction and found that the expression of FGFR4 gradually declined (Fig. 2B), which is in agreement with the finding that FGFR4 is excluded from the neuroectoderm of mouse embryos [20]. In contrast, FGFR1, FGFR2, and FGFR3 expressions were significantly increased during the conversion of ES 


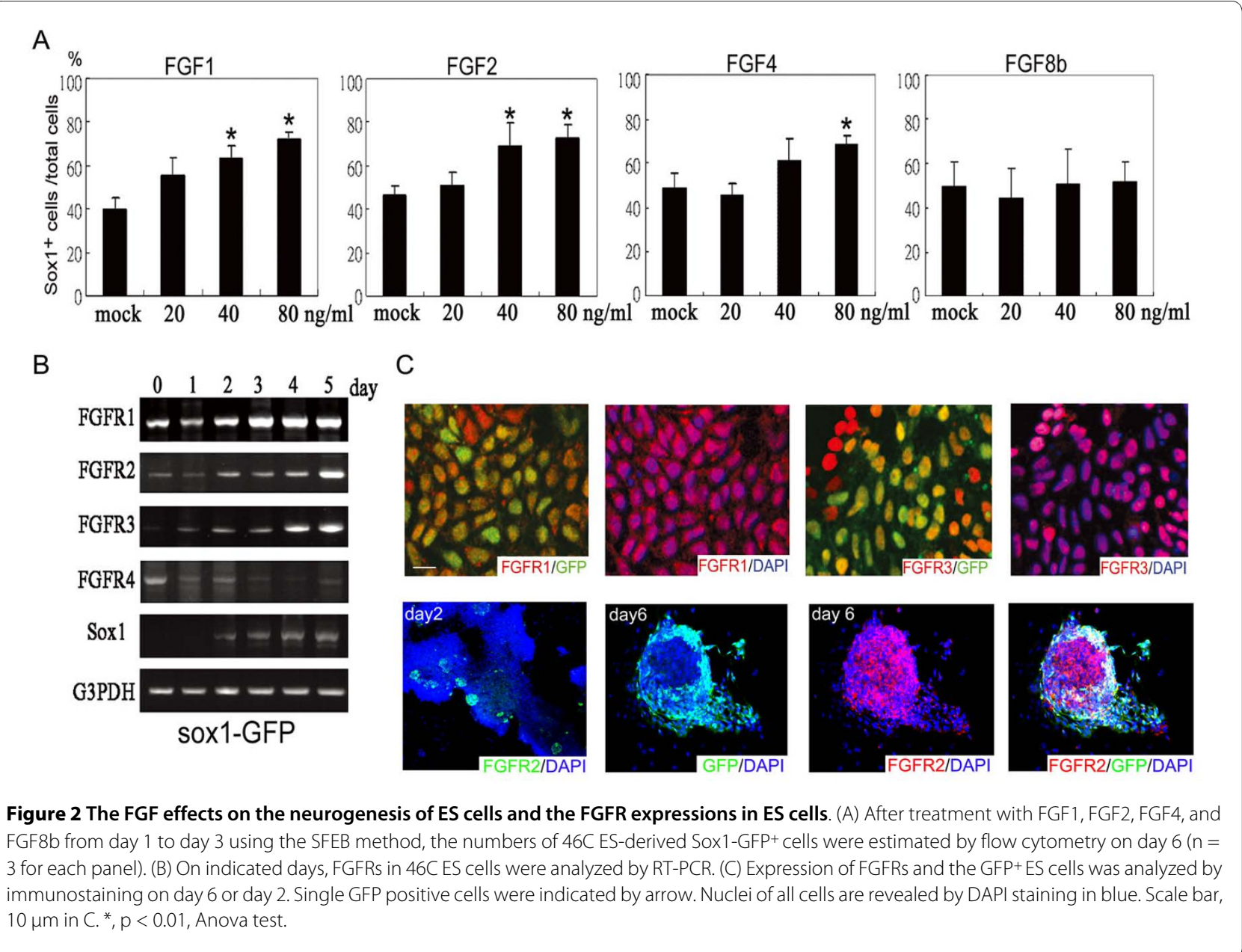

into neuroblast cells. Immunocytostaining revealed that both FGFR1 and FGFR3 were detected in cytosol and nuclei in neural derivatives (Fig. 2C). On day 6, GFP+ signals were colocalized with FGFR1- and FGFR3-expressing cells, suggesting that both signals may be involved in neurogenesis (Fig. 2C). RT-PCR and immunostaining, shown in Figs. $2 \mathrm{~B}$ and $2 \mathrm{C}$, indicated that the expression of FGFR2 in differentiating ES cells was robustly induced and was localized on the cell membrane and cytosol, rather than in the nucleus. We also found that FGFR2 was not completely coexpressed with the GFP in 46C cells on day 6 (Fig. 2C), suggesting that FGFR2 is involved in the formation of subtypes of neurons. Taken together, these results suggest that FGFR1 and FGFR3 are generally required for neural induction and FGF8b is incompetent on the enhancement of neurogenesis of ES cells.

\section{Neural induction enhanced by FGF was not mediated through the anti-apoptosis or cell proliferation on Sox ${ }^{+}$ cells}

We treated 46C ES cells with or without FGF1 from day 1 through day 3 and detect the Sox $1-\mathrm{GFP}^{+}$cells from day 1 to day 8 (Fig. 3A). The number of Sox $1^{+}$cells became $20 \%$ of total cells on day 3 and reached the plateau, $50 \%$ of total cells, on day 7. Treatment of FGF1 consistently and dose-dependently enhanced the neurogenesis on day 3 through day 7. We also found that FGF treatment can promote but cannot shorten the time of the neural induction from ES cells. The Sox1-GFP+ cells did not appear on differentiation day 2, regardless of the FGF1 treatment.

The increase of Sox $1^{+}$cells in the FGF1-treated condition may result from enhanced proliferation and/or reduced apoptosis of neuroblast cells. To test these possibilities, FGF1 was incubated with the $46 \mathrm{C}$ cells, and the apoptosis and proliferation of Sox $1^{+}$cells were analyzed by staining of activated caspase- 3 and Ki67, respectively. Double staining of cleaved caspase- 3 and GFP revealed that less than $5 \%$ double positive cells were detected (Fig. 3B). Similar results were obtained in FGF1-treated Sox $1^{+}$ cells (data not shown). The percentages of $\mathrm{Ki}^{+} 7^{+}$cells in Sox $1^{+}$population were $24.75 \%$ (196/792) and $25.48 \%$ (362/1421) in SFEB- and SFEB/FGF1-treated cells respectively (Fig. 3C and 3D), demonstrating that FGF-triggered 

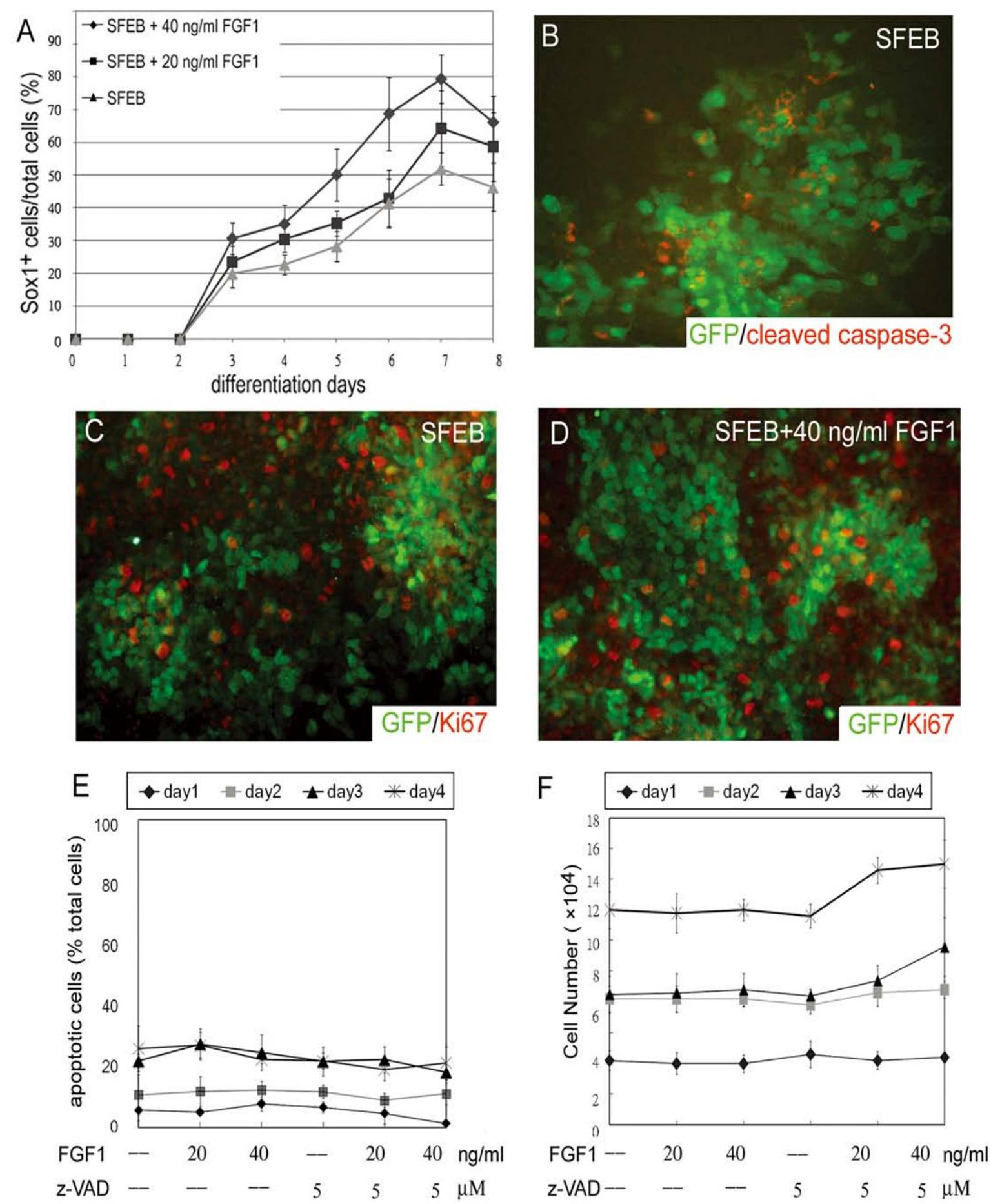

Figure 3 The apoptosis and the proliferation on committed neuroblast cells. (A) The induction of Sox1-GFP+ cells from 46C cells were detected by flow cytometry under the SFEB and SFEB/FGF1 condition. (B) The differentiating ES cells were labeled with cleaved caspase-3 (red), which detects the cleaved fragment of caspase-3 (17/19 kDa), in Sox1/GFP+ cells on differentiating day 4. (C, D) Proliferating GFP+ cells were marked with the nuclear staining of ki67 on day 4. (E) Total apoptotic cells, characterized with Annexin-V labeling, were estimated by flow cytometry after FGF and/or z-VADfmk, a membrane-permeable pan-caspase inhibitor, from day 1 to day 4. Culture media were changed every day. (F) Total cell numbers were counted in triplicate using trypan blue exclusion at indicated times. 
neurogenesis may not mediated through the enhancement of Sox1 cell proliferation.

We also found that on day 1 through day 4, the total number of apoptotic cells was not reduced after treatment with $40 \mathrm{ng} / \mathrm{ml} \mathrm{FGF1}$, or with $5 \mu \mathrm{M}$ of a pan-caspase inhibitor, z-VAD-fmk. Even after the addition of both FGF1 and z-VAD-fmk, the rescue of apoptotic cells was not significant (Fig. 3E). The total ES cell population was also counted on differentiation days 1 to 4 . No statistical significance in number was seen after treatment with FGF1 and/or z-VAD-fmk (Fig. 3F). In sum, these results suggest that the FGF-steering neurogenesis mainly depends on the enforcing differentiation of ES cells, rather than on anti-apoptosis or cell proliferation.

\section{Neural induction of ES cells was mediated through the activation of MAPK pathways}

Given that phosphorylated intracellular domains of FGFRs activate downstream phosphoinositide-3 kinase (PI3K)/AKT and three major serine/threonine MAPKs, including ERK 1/2, JNKs, and p38 kinases, we further investigated which MAPK pathways were responsible for the FGF-dependent neural induction. We found that single suspended ES cells continued to initiate phosphorylated JNK during differentiation (Fig. 4A). Significant enhancement of ERK activation was observed in $20 \mathrm{ng} / \mathrm{ml}$ FGF1-treated ES cells, providing the linkage of biochemical evidences of FGF signal with its pro-neural function. FGF1 promoted the AKT phosphorylation and the activities of all three MAPKs in differentiating ES cells at $12 \mathrm{hr}$ differentiation (Fig. 4B). Immunoblotting showed that the total amount of AKT, JNK, p38 MAPK, and ERK1/2 protein expression was not altered between control and SFEB conditions. Especially, JNK1 and ERK2 were the major phosphorylated isoforms of JNKs and ERKs in the differentiating ES cells, respectively.

Specific pharmacological inhibitors of MAPKs, shown affecting their respective kinase targets in Fig. 4B, were administrated to delineate the kinases involved in neurogenesis. We found that a PI3K/AKT inhibitor, LY294002, significantly reduced the formation of Sox1-GFP+ ${ }^{+}$cells under SFEB and SFEB/FGF1 conditions (Fig. 4C and 4D).

Intriguingly, a JNK inhibitor and an ERK inhibitor, SP600125 and U0126, respectively, dramatically blocked the neural formation of ES cells and abolished the FGFmediated neurogenesis (Fig. 4C and 4D). Nevertheless, there was no significant reduction of Sox1-GFP+ cells after treatment with p38 kinase inhibitor, in both exogenous FGF present or absent condition (Fig. 4C and 4D). In addition, to verify the role of JNK isotypes in neural differentiation of ES cells, stable clones expressing the JNK1 and JNK2 dominant negative mutants (JNK1a1apf and JNK2a2apf) were established (Fig. 5A and 5B). We found that specific inhibition of JNK1, but not JNK2, sig- nificantly reduced the formation of Sox $1^{+}$and N-cadherin $^{+}$cells (Fig. 5C, 5D and 5E), indicating that JNK1 is essential for the neural induction of ES cells.

\section{Response-time windows for the FGF-mediated neurogenesis}

To verify the FGF response windows during ES differentiation, $40 \mathrm{ng} / \mathrm{ml} \mathrm{FGF1}$ was incubated with $46 \mathrm{C}$ cells for 24 hr on individual day 1 to 4 (Fig. 6A). ES-derived neural cells were analyzed on day 6 by FACS. FGF1 treatment in the first $24 \mathrm{hr}$ window was sufficient to promote Sox 1 cell induction (Fig. 6B, the lane D1). Neurogenic effects were also observed when the ES cells were incubated with FGF1 on day 2 or 3 (Fig. 6B, the lane D2 and D3). This result argues that transient FGF activation is sufficient to enforce early cell-fate commitment and neural induction of ES cells. In contrast, JNK and ERK inhibitors caused only a short-term reduction of neurogenesis and a delay in commitment. As shown in Figs. 6C and 6D, neural inhibition was observed on day 6 when MAPK signals were constantly depressed throughout days 1 to 3 (Fig. 6D; the lane D1-3). Transient treatments of both inhibitors on individual days did not show the suppression of neural induction (Fig. 6D; the lane D1, D2 and D3). Interestingly, we also found that $\mathrm{GFP}^{+}$cell population with the treatment of MAPK inhibitors throughout days 1 to 3 gradually increased from $26 \pm 5.5 \%$ on day 6 to $55 \pm 6.7 \%$ of total cells on day 9 (data not shown), suggesting that inhibition of JNK and ERK retards the ES cell commitment, rather than promotes non-neural lineages.

\section{Cell lineages of the ES cells treated with MAPK inhibitors}

Reduction of the neural induction by the JNK and ERK inhibitors could be caused by the increased undifferentiating ES cells or non-neural lineages. In this study, we demonstrated that inactivation of both JNK and ERK enhanced the expression of pluripotent markers Oct4 and Nanog in differentiating ES cells on day 6 (Figs. 7A and $7 \mathrm{~B}$ ), indicating that both phosphorylated JNK and ERK are negative regulators of self-renewal of ES cells. It is recently documented that ERK2 null ES cells fail to commit into neural and mesodermal cells [21-24]. Similarly, rare brachyury $(\mathrm{T})$ expressed cells were found in SP600125- and U0126-treated ES cells, compared to 5.2 $\pm 0.2 \%$ brachyury-positive cells in the total population under SFEB (Fig. 7C and 7E). The Sox $17^{+}$cells, representing endoderm of differentiating ES cells, only showed less $5 \%$ of total ES cells on day 6 under the SFEB condition (Fig. 7D). No significant elevation of Sox17+ cells was observed in JNK/ERK inhibitors treated ES cells (Fig. 7F). In addition, we also did not find the appearance of cytokeratin 14 (K14) positive cells, representing the epidermal precursor cells, in the SFEB-differentiating ES cells even after the treatment of MAPK 
A
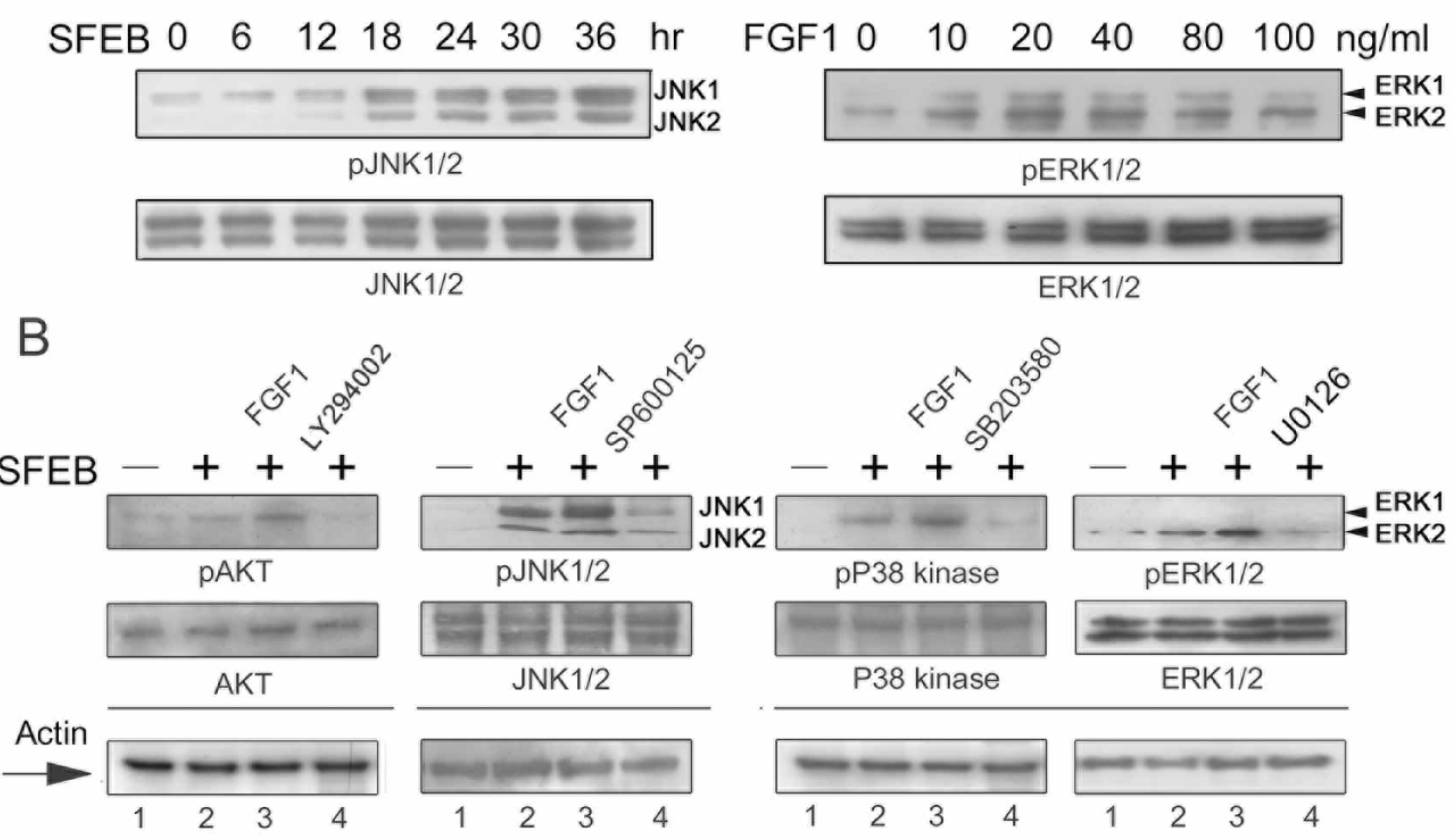

\section{SFEB/inhibitors}
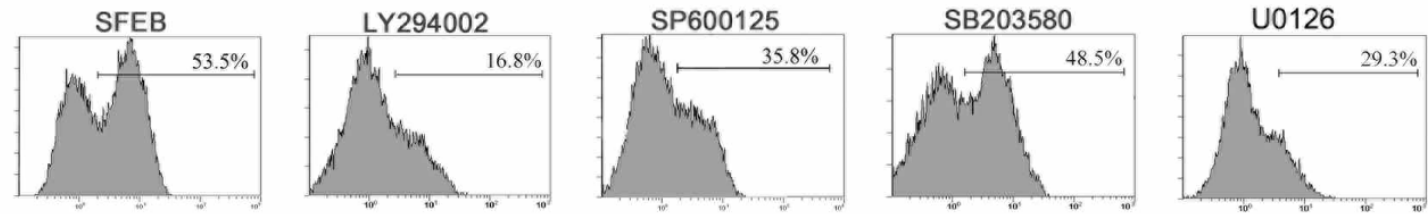

\section{SFEB/FGF1+inhibitors}
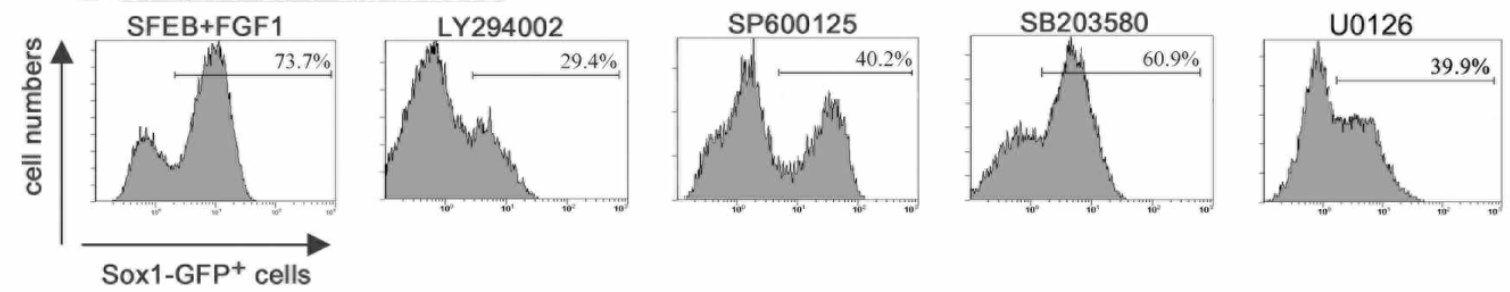

Figure 4 Effects of MAPK inhibitors on neural induction of ES cells. (A) Total cell lysates were collected from differentiating ES cells at indicated times under SFEB condition. Kinetic JNKs activation was analyzed by western blot. FGF1 dose-effect on differentiating ES cells was revealed by ERK phosphorylation at 30 min differentiation. (B) Downstream FGF signals were further detected with individual specific antibodies at 12 hr post-treat-

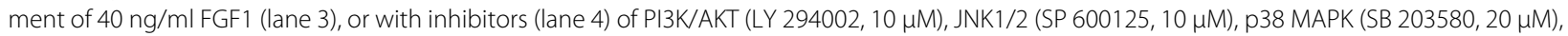
and ERK1/2 (U0126, 5 MM). After treatment with the inhibitors (C) or FGF1 $(40 \mathrm{ng} / \mathrm{ml})$ plus the inhibitors (D) from day 1 to day 3, the derived cells were collected for FACS analysis on day 6 . The same concentrations of reagents were applied in these experiments. Representative results were shown from experiments done at least in triplicate.

inhibitors. These results demonstrated that the reduction of neural formation by the inactivation of MAPK was caused by the blockage of ES differentiation, rather than by the enhancement of formation of mesoendodermal nor epidermal lineages.

\section{Discussion}

Neural induction requires sequential signals to direct uncommitted ectoderm into the definitive neural plate [25]. Cumulative evidence supports the fact that FGF is an essential factor for neurogenesis [26,27]. Interestingly, activation of the Ras/MAPK pathway, rather than the 
A

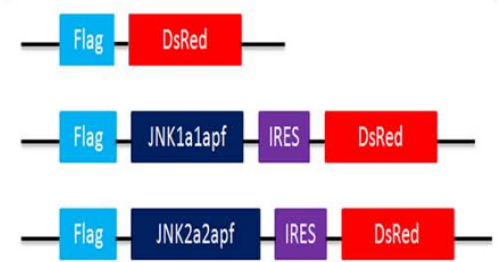

B

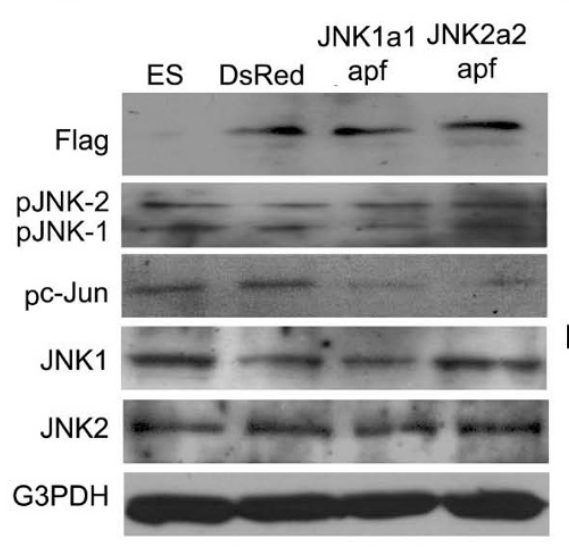

$\mathrm{C}$

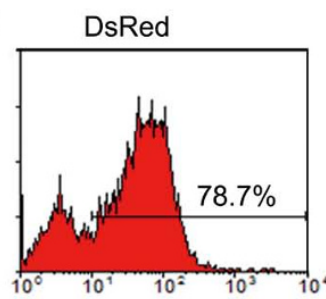

D

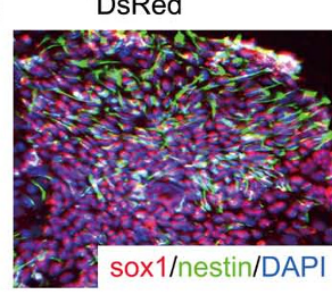

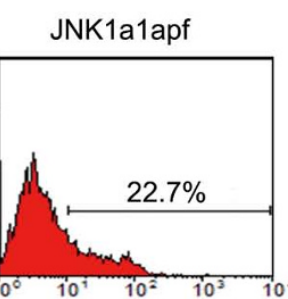

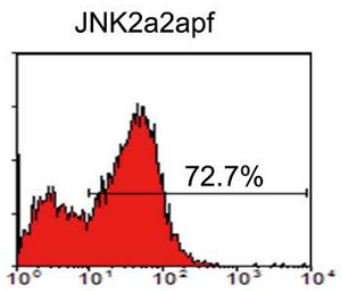

JNK1a1apf
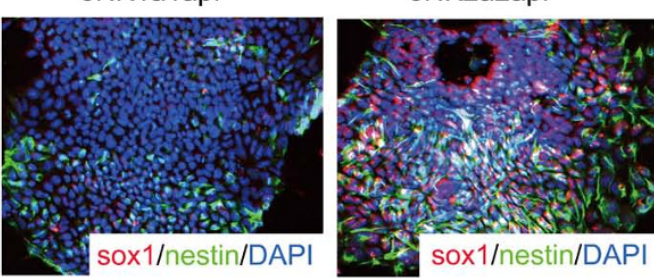

E
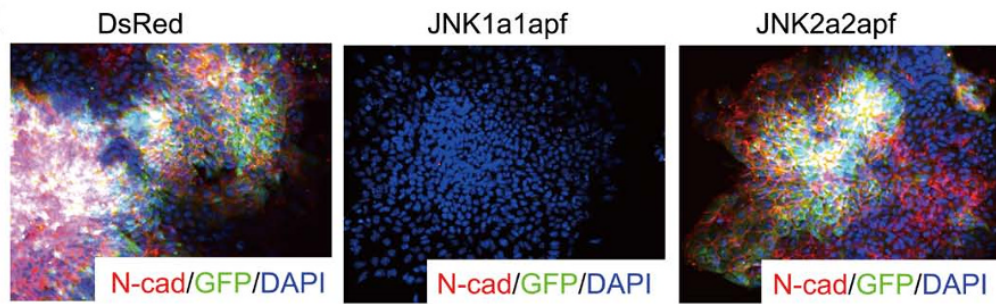

Figure 5 Genetic inhibition of JNKs in differentiating ES cells. (A) Flag-tagged dominant-negative mutants of JNK1 and JNK2 (JNK1a1-apf and JNK2a2-apf) were conjugated with IRES-DsRed for the tracing of the consistently expressing cells. (B) The expression of flag, phosphorylated JNKs, phosphorylated c-Jun (pc-Jun) and total amount of JNK1 and JNK2 were revealed by western blot. (C) Their efficiencies of neural formation were estimated by FACS analyses. The expressions of neural markers are also examined, such as Sox1 (D), nestin (D) and N-cadherin (N-cad) (E).

A

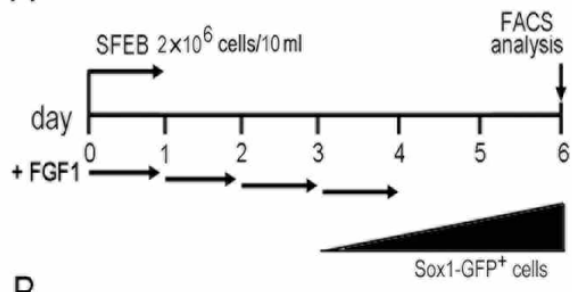

B

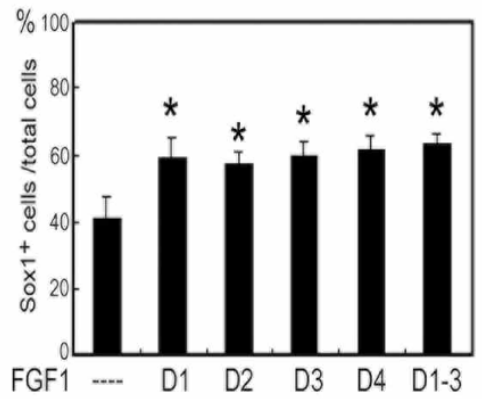

C

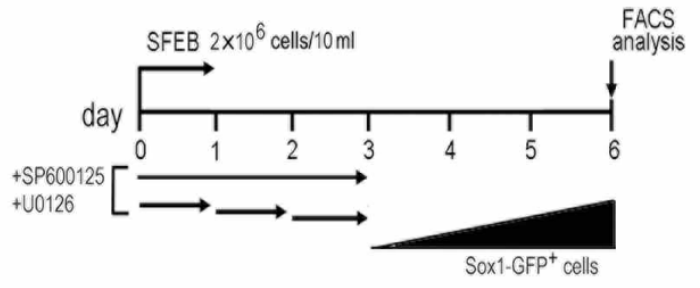

D

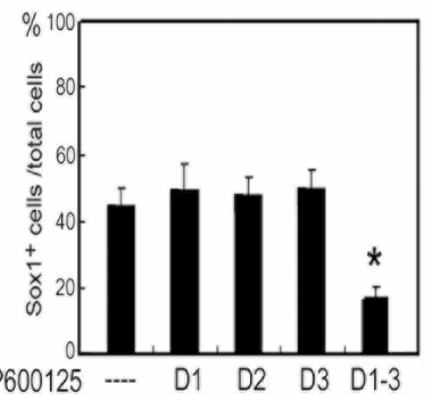

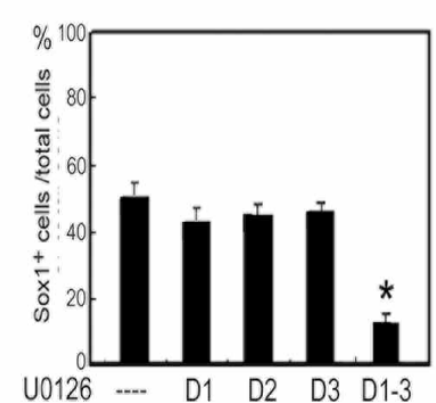

Figure 6 Response windows of FGF and MAPK inhibitors in differentiating ES cells. (A) FGF1 at $40 \mathrm{ng} / \mathrm{ml}$ was applied to $46 C$ ES cells on individual days (D1, D2, D3, D4) or from day 1 through 4 (D1-4). (B) Derived GFP+ cells were analyzed by FACS on day 6 . Independent experiments done in triplicate are illustrated. (C) As the indicated experimental conditions, the induction of Sox1-GFP+ cells on day 6 was shown in (D) after FACS analysis. SP600125 and U0126, $10 \mu \mathrm{M}$ and $5 \mu \mathrm{M}$, respectively. 
A

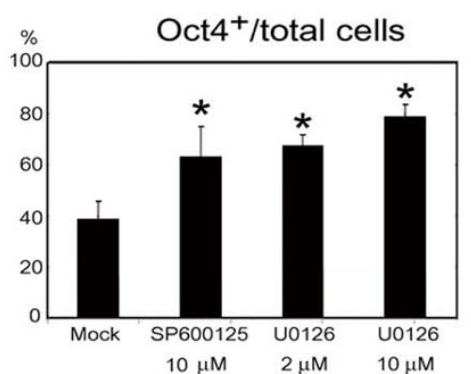

B

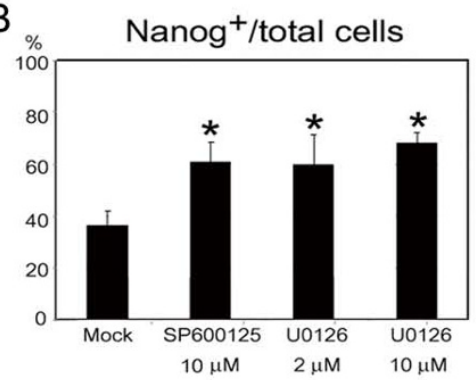

C

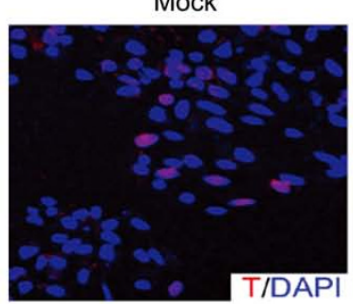

D

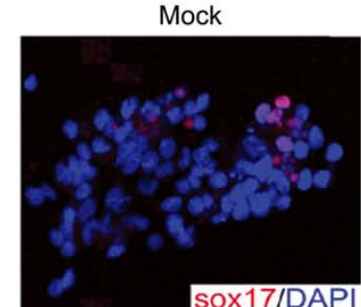

E

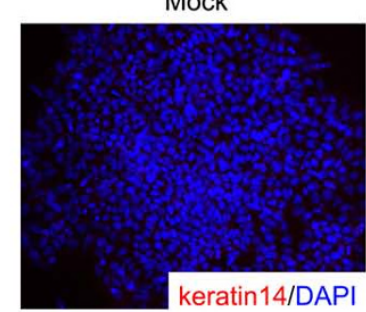

$\mathrm{F}$

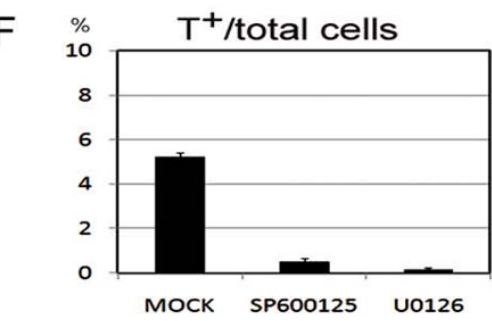

SP600125

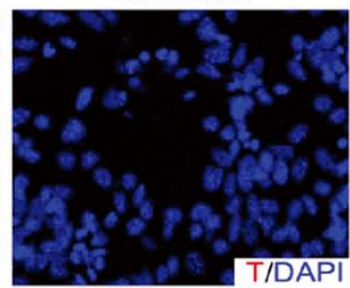

SP600125

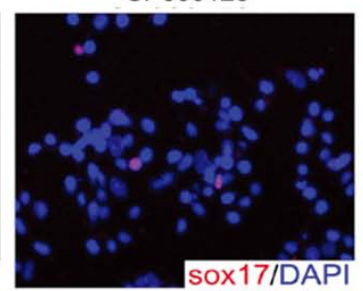

SP600125
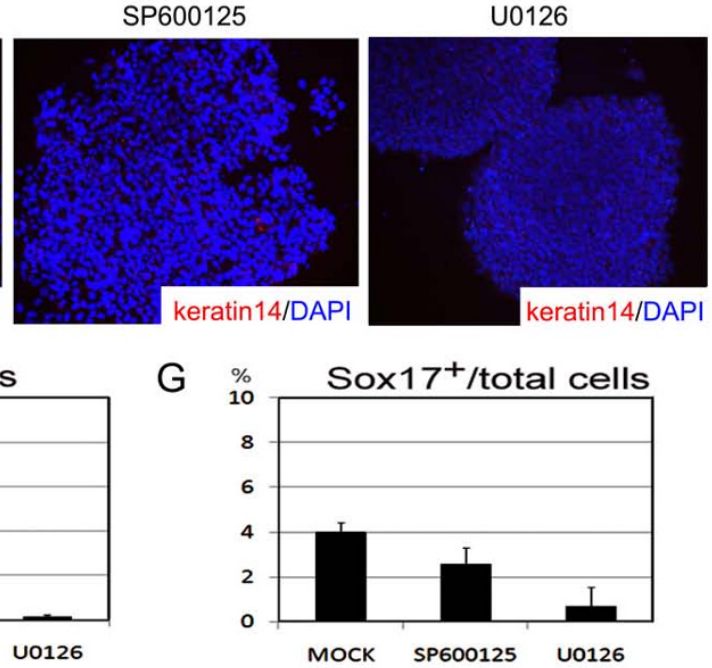

Figure 7 Both inhibitors of JNK and ERK retarded ES differentiation. After treatment with $10 \mu \mathrm{M}$ SP600125, 2 or $10 \mu \mathrm{M}$ U0126 from days 1-3, ES cells were plated on $0.1 \%$ matrigel-coated glasses and stained with anti-Oct4 (A) and anti-Nanog antibodies (B) on day 6 . The ratio of undifferentiated pluripotent ES cells to total DAPI+ cells ( $n>500$ cells) was estimated from experiments done in triplicate. Brachyury (T) (C), Sox17 (D) and cytokeratin 14 (E) expressions, representing mesodermal, endodermal and surface ectodermal cell lineages respectively, were examined in ES cells on day 6 with SFEB treatment. Nuclei of all cells are seen by DAPI staining in blue. The statistic results of the cell numbers in panel $C$ and $D$ were also estimated, respectively $(\mathrm{E}, \mathrm{F})$. 
diluted BMP ligands, has been shown to be responsible for the neural cell fate of the fully dissociated animal cap cells, arguing against the simplistic neural default model [5]. The primitive streak- or organizer-derived BMP inhibitors are not the only signals required for neurogenesis. FGF and the other developmental cues, such as Wnt and Notch, also participate in neural induction in a sophisticated manner [25].

It is noteworthy to emphasize that the activation of MAPK during ES differentiation may not solely depend on FGFR signals and other neural instructing factors could also contribute to the neural induction through JNK or ERK activation, such as insulin-like growth factor (IGF) [28]. Treatment of JNK and ERK inhibitors should simultaneously abolish the endogenous receptor tyrosine kinase signals of differentiating ES cells. Here we showed that neural induction of ES cells was accompanied with the elevated expression of FGFRs and the activation of MAPK pathway (Figs. 2B, 4A and 4B). Pharmacological evidences (Fig. 4C) further supported that differentiation into primitive neuroepithelial cells relied on the activation of both JNK and ERK pathways, but not the p38 MAPK pathway (Fig. 4C). Exogenous FGF-triggered neurogenesis was completely reduced by the JNK and ERK inhibitors (Fig. 4D). Taken together, these data highlights the importance of FGFR activation and of individual MAPK signals in the ES-neuron conversion.

Both pharmacological and genetic evidences support the important role of JNK1 for the neural induction of ES cells (Fig. 4C, D and 5). These results are consistent with the previous finding that JNK1 ${ }^{-/}$ES cell has a significant reduction in RA-triggered neurogenesis and that JNK/ Stress-associated activated protein 1 (JSAP1) is involved in early embryonic neurogenesis $[29,30]$. While a neural tube defect is only observed in JNK1/JNK2 doubleknockout mice and a JNK1 and JNK2 single-null embryo is normal [31]. It is important to further explore the reason of discrepancy between in vitro and in vivo data and the JNK regulatory networks which participate in neural fate decision and the development of primitive neuroectoderm.

Genetic manipulation has shown that ERK1-null mice are healthy after birth, whereas disruption of the ERK2 gene results in abnormal trophectodermal and mesodermal development $[32,33]$. In vitro ES differentiation has also revealed that inhibition of ERK2 completely blocks neural and mesodermal formation, suggesting that ERK2 is essential for the initiation of cell fate commitment of epiblast cells $[21,24]$. In this study, we showed that inhibition of MAPK signals sustained the undifferentiated status and the expression of pluripotent markers under the SFEB condition. In future studies, it will be important to understand how the regulatory networks of MAPKs are affected after deprivation of LIF and how they initiate somatic cell induction in ES cells.

\section{Conclusions}

Based on a simple and efficient neural induction method, we demonstrate that FGF-triggered neurogenesis of ES cells is not involved in cell proliferation or inhibition of apoptosis. Activation of the ERK2 and JNK1 pathways, rather than p38 MAP kinase, is mainly responsible for the neural induction of ES cells. Release of pharmacological inhibition re-initiated the ES differentiation and neurogenesis, indicating that the FGF pathway participates in the initiation of ES commitment into embryonic cell lineages.

\section{List of abbreviations}

ESC: embryonic stem cell; FGF: fibroblast growth factor; MAPK: mitogen-activated protein kinase; SFEB: serumfree embryoid body-like formation.

\section{Competing interests}

The authors declare that they have no competing interests.

\section{Authors' contributions}

CWC, SCS, HCP and HLS carried out the neural differentiation and drafted the manuscript. KHL provided the mES cells and participated in the design of the study. CSL, IMC SZL and HLS participated in the design of the study and performed the statistical analysis. All authors read and approved the final manuscript.

\section{Acknowledgements}

This work was supported by the Changhua Christian Hospital (C.S.L.), National Health Research Institutes (H.L.S.) as well as the National Science Council (H.L.S.) of Taiwan. This work was also granted from the Taichung Veterans General Hospital and National Chung Hsing University (TCVGH-NCHU-9776614 and -977602; to H.L.S and H.C.P.), Taichung, Taiwan. We also thank for the support from the core laboratory of tissue engineering and stem cells center in $\mathrm{NCHU}$.

\section{Author Details}

'Department of Life Sciences, National Chung-Hsing University, Taichung, Taiwan, ${ }^{2}$ Department of Medical Research, Changhua Christian Hospital, Changhua, Taiwan, 3 Institute of Cellular and Systems Medicine, National Health Research Institutes; Miaoli, Taiwan, ${ }^{4}$ Department of Neurosurgery, Taichung Veterans General Hospital; Taichung, Taiwan, ${ }^{5}$ Animal Technology Institute Taiwan; Miaoli, Taiwan, ${ }^{6}$ Center for Neuropsychiatry, China Medical University and Hospital, Taichung, Taiwan; China Medical University Beigang Hospital, Yunlin, Taiwan; Department of Immunology, China Medical University, Taichung, Taiwan and 7 Department of Physical Therapy, China Medical University, Taichung, Taiwan

Received: 28 December 2009 Accepted: 29 April 2010 Published: 29 April 2010

\section{References}

1. Streit A, Berliner AJ, Papanayotou C, Sirulnik A, Stern CD: Initiation of neural induction by FGF signalling before gastrulation. Nature 2000 406:74-78.

2. Streit A, Stern CD: Establishment and maintenance of the border of the neural plate in the chick: involvement of FGF and BMP activity. Mech Dev 1999, 82:51-66.

3. Linker C, Stern CD: Neural induction requires BMP inhibition only as a late step, and involves signals other than FGF and Wnt antagonists. Development 2004, 131:5671-5681. 
4. Sheng G, dos Reis M, Stern CD: Churchill, a zinc finger transcriptional activator, regulates the transition between gastrulation and neurulation. Cell 2003, 115:603-613.

5. Kuroda H, Fuentealba L, Ikeda A, Reversade B, De Robertis EM: Default neural induction: neuralization of dissociated Xenopus cells is mediated by Ras/MAPK activation. Genes Dev 2005, 19:1022-1027.

6. Pera MF, Trounson AO: Human embryonic stem cells: prospects for development. Development 2004, 131:5515-5525.

7. Spagnoli FM, Hemmati-Brivanlou A: Guiding embryonic stem cells towards differentiation: lessons from molecular embryology. Curr Opin Genet Dev 2006, 16:469-475.

8. Kawasaki H, Mizuseki K, Nishikawa S, Kaneko S, Kuwana Y, Nakanishi S, Nishikawa SI, Sasai Y: Induction of midbrain dopaminergic neurons from ES cells by stromal cell-derived inducing activity. Neuron 2000, 28:31-40.

9. Wichterle H, Lieberam I, Porter JA, Jessell TM: Directed differentiation of embryonic stem cells into motor neurons. Cell 2002, 110:385-397.

10. Mizuseki K, Sakamoto T, Watanabe K, Muguruma K, Ikeya M, Nishiyama A, Arakawa A, Suemori H, Nakatsuji N, Kawasaki H, Murakami F, Sasai Y: Generation of neural crest-derived peripheral neurons and floor plate cells from mouse and primate embryonic stem cells. Proc Nat/ Acad Sci USA 2003, 100:5828-5833.

11. Kohga H, Obata K: Retinoic acid-induced neural tube defects with multiple canals in the chick: immunohistochemistry with monoclonal antibodies. Neurosci Res 1992, 13:175-187.

12. Perrier AL, Tabar V, Barberi T, Rubio ME, Bruses J, Topf N, Harrison NL, Studer $\mathrm{L}$ : Derivation of midbrain dopamine neurons from human embryonic stem cells. Proc Natl Acad Sci USA 2004, 101:12543-12548,

13. Ying QL, Stavridis M, Griffiths D, Li M, Smith A: Conversion of embryonic stem cells into neuroectodermal precursors in adherent monoculture. Nat Biotechnol 2003, 21:183-186.

14. Aubert J, Stavridis MP, Tweedie S, O'Reilly M, Vierlinger K, Li M, Ghazal P, Pratt T, Mason JO, Roy D, Smith A: Screening for mammalian neural genes via fluorescence-activated cell sorter purification of neural precursors from Sox1-gfp knock-in mice. Proc Natl Acad Sci USA 2003, 100:11836-11841

15. Watanabe K, Kamiya D, Nishiyama A, Katayama T, Nozaki S, Kawasaki H, Watanabe Y, Mizuseki K, Sasai Y: Directed differentiation of telencephalic precursors from embryonic stem cells. Nat Neurosci 2005, 8:288-296.

16. Lee KH, Chuang CK, Wang HW, Stone L, Chen CH, Tu CF: An alternative simple method for mass production of chimeric embryos by coculturing denuded embryos and embryonic stem cells in Eppendorf vials. Theriogenology 2007, 67:228-237.

17. Patrie KM, Botelho MJ, Franklin K, Chiu IM: Site-directed mutagenesis and molecular modeling identify a crucial amino acid in specifying the heparin affinity of FGF-1. Biochemistry 1999, 38:9264-9272.

18. Wojtaszek PA, Heasley LE, Siriwardana G, Berl T: Dominant-negative c-Jun $\mathrm{NH} 2$-terminal kinase 2 sensitizes renal inner medullary collecting duct cells to hypertonicity-induced lethality independent of organic osmolyte transport. J Biol Chem 1998, 273:800-804.

19. Xiao L, Lang W: A dominant role for the c-Jun NH2-terminal kinase in oncogenic ras-induced morphologic transformation of human lung carcinoma cells. Cancer research 2000, 60:400-408

20. Stark KL, McMahon JA, McMahon AP: FGFR-4, a new member of the fibroblast growth factor receptor family, expressed in the definitive endoderm and skeletal muscle lineages of the mouse. Development 1991, 113:641-651.

21. Kunath T, Saba-El-Leil MK, Almousailleakh M, Wray J, Meloche S, Smith A: FGF stimulation of the Erk $1 / 2$ signalling cascade triggers transition of pluripotent embryonic stem cells from self-renewal to lineage commitment. Development 2007, 134:2895-2902.

22. Burdon T, Stracey C, Chambers I, Nichols J, Smith A: Suppression of SHP-2 and ERK signalling promotes self-renewal of mouse embryonic stem cells. Dev Biol 1999, 210:30-43.

23. Li Z, Theus MH, Wei L: Role of ERK $1 / 2$ signaling in neuronal differentiation of cultured embryonic stem cells. Dev Growth Differ 2006, 48:513-523.

24. Stavridis MP, Lunn JS, Collins BJ, Storey KG: A discrete period of FGFinduced Erk $1 / 2$ signalling is required for vertebrate neural specification. Development 2007, 134:2889-2894.

25. Stern CD: Neural induction: 10 years on since the 'default model'. Curr Opin Cell Biol 2006, 18:692-697.
26. Delaune E, Lemaire P, Kodjabachian L: Neural induction in Xenopus requires early FGF signalling in addition to BMP inhibition. Development 2005, 132:299-310

27. Wilson SI, Graziano E, Harland R, Jessell TM, Edlund T: An early requirement for FGF signalling in the acquisition of neural cell fate in the chick embryo. Curr Biol 2000, 10:421-429.

28. De Robertis EM, Kuroda H: Dorsal-ventral patterning and neural induction in Xenopus embryos. Annu Rev Cell Dev Biol 2004, 20:285-308.

29. Xu P, Yoshioka K, Yoshimura D, Tominaga Y, Nishioka T, Ito M, Nakabeppu $Y$ : In vitro development of mouse embryonic stem cells lacking JNK/ stress-activated protein kinase-associated protein 1 (JSAP1) scaffold protein revealed its requirement during early embryonic neurogenesis. J Biol Chem 2003, 278:48422-48433.

30. Amura CR, Marek L, Winn RA, Heasley LE: Inhibited neurogenesis in JNK1-deficient embryonic stem cells. Mol Cell Biol 2005, 25:10791-10802.

31. Kuan CY, Yang DD, Samanta Roy DR, Davis RJ, Rakic P, Flavell RA: The Jnk1 and Jnk2 protein kinases are required for regional specific apoptosis during early brain development. Neuron 1999, 22:667-676.

32. Pages $G$, Guerin $S$, Grall D, Bonino F, Smith A, Anjuere F, Auberger $P$, Pouyssegur J: Defective thymocyte maturation in p44 MAP kinase (Erk 1) knockout mice. Science 1999, 286:1374-1377.

33. Yao Y, Li W, Wu J, Germann UA, Su MS, Kuida K, Boucher DM: Extracellular signal-regulated kinase 2 is necessary for mesoderm differentiation. Proc Natl Acad Sci USA 2003, 100:12759-12764.

doi: $10.1186 / 1423-0127-17-33$

Cite this article as: Chen et al., The signals of FGFs on the neurogenesis of embryonic stem cells Journal of Biomedical Science 2010, 17:33

\section{Submit your next manuscript to BioMed Central and take full advantage of:}

- Convenient online submission

- Thorough peer review

- No space constraints or color figure charges

- Immediate publication on acceptance

- Inclusion in PubMed, CAS, Scopus and Google Scholar

- Research which is freely available for redistribution

Submit your manuscript at www.biomedcentral.com/submit
C) Biomed Central 